Article

\title{
Simulation of mTBI Utilizing White Matter Properties from MRE
}

Amit Madhukar ${ }^{1}$ and Martin Ostoja-Starzewski ${ }^{1,2}$

1 Department of Mechanical Science \& Engineering, University of Illinois at Urbana-Champaign, Urbana, IL 61801, U.S.A.

2 Beckman Institute and Institute for Condensed Matter Theory, University of Illinois at Urbana-Champaign, Urbana, IL, 61801, U.S.A.

\begin{abstract}
We extend our high-resolution MRI-based Finite Element (FE) head model, previously presented and validated in [1-3], by considering the heterogeneities of the white matter structures captured through the use of Magnetic Resonance Elastography (MRE). This approach imparts more sophistication to our numerical model and yields results that more closely match experimental results. It is found that the peak pressure more closely matches the experiments as compared to the heterogeneous case. Qualitatively, we find differences in stress wave propagation near the corpus callosum and the corona radiata, which are stiffer on average than the global white matter. We are able to study the effects of these stiff structures on transient stress wave propagation within the cerebrum, something that cannot be done with a homogenized material model.
\end{abstract}

\section{Introduction}

Traumatic brain injury (TBI) commonly occurs by impacts - direct blows to the head; or by impulse - a sudden movement of the head, especially prevalent in car crashes. TBI remains a pressing concern for study with approximately 2.8 million cases of TBI-related injuries reported in the United States [4]. Clinical studies have shown that damage to the brain in blunt head injuries is primarily confined to the cerebral white matter, with a propensity for lesions in the brainstem and the corpus callosum, i.e., regions with highly organized axon tracts. [5-10]. These regions serve as vital connection points to other parts of the brain, meaning damage to them is potentially more dangerous. In this work we introduce a heterogeneous material description of white matter structures to our high-resolution Finite Element (FE) model to account for the local differences in mechanical response between different regions of the brain.

The FE method is commonly used to determine the mechanical response of brain tissue in order to develop improved diagnostic tools and protective measures to reduce the prevalence of TBI. The 
accuracy of these FE models are highly dependent on the accuracy of the material model. To date most finite element models have utilized mechanical properties averaged over large portions of the brain, as reviewed in [11], thus ignoring potentially significant effects of local structures. In actuality, the tissues of the brain are heterogeneous, their constitutive response varying from location to location. This is most noticeable for white matter due to the presence of axons with diverse orientations. Overall, the shear stiffness of white matter is 1.2-2.6 higher than that of gray matter [12]. Locally, white matter tracts with highly oriented fibers such as the corpus callosum and corona radiata have material properties vastly different from other regions. Johnson et al. [13] determined that global white matter was softer on average than either the corpus callosum or the corona radiata. This can be explained by considering the structure of these regions. The corpus callosum is a tight bundle of highly aligned fibers which is expected to provide more structural rigidity than the superficial white matter. The fan-like structure of the corona radiata provides a similar response, though to a lesser extent since the fibers are not as highly aligned. As such the corpus callosum was found to be approximately $11 \%$ stiffer than the corona radiata. The brainstem is another structure with a high level of heterogeneity. Arbogast and Margulies [10] investigated the prevalence of trauma observed in the brainstem after head injuries. They determined that the brainstem was $80-100 \%$ stiffer than the cerebrum and concluded this regional stiffness variation is one reason for the selective vulnerability of this region to rotational motion. FE models that utilize homogenized white matter material properties have no way to resolve these local features.

A very useful tool to measure heterogeneity in-vivo is Magnetic Resonance Elastography (MRE) [14] where the head is excited with shear waves to quantitatively assess the local mechanical properties of brain tissue. This technique has been successfully applied to a variety of different applications such as decrease in whole-brain stiffness with age [15] and as a result of certain neurodegenerative diseases [16]; measurement of tumor stiffness [17]; and as a marker for TBI severity [18]. MRE is applied as a three-step process beginning by first inducing shear waves in tissue with frequencies ranging from 50-500 Hz using an external driver. Second, the waves are imaged using a phase-contrast MRI pulse sequence synchronized with the frequency of the applied vibration. Finally, the mechanical properties of the tissue are estimated by inverting the observed displacements using a viscoelastic material model; such as that presented by Van Houten et al. [19].

In this work, we utilize our previously homogeneous FE model - presented and validated in our previous works [1-3] - and introduce a voxel-based heterogeneous material model using results from Johnson et al. [13]. In order to properly resolve the high-frequency waves generated during such vibrations, the finite element mesh must be properly refined, as argued in [11]. The mechanical properties used in this work are reconstructed at the same spatial resolution as the displacement data 
captured during the MRE process. This in turn generates a finite element mesh that has more than sufficient resolution to accurately capture the dynamic shear wave propagation during impacts. To the best of our knowledge, this is the first attempt to include heterogeneity of brain tissue via MRE in a high-resolution FE model.

\section{Model Formulation}

\subsection{MRE Aquisiton and Inversion}

The heterogeneous properties of the white matter are taken from the work by Johnson et al. [13]. A brief overview of the acquisition and inversion is presented here. A more detailed discussion of this process can be found in [20]. Shear waves are generated at a frequency of $50 \mathrm{~Hz}$ by placing the subject's head on a custom cradle attached to an electromagnetic shaker via a rigid rod. The actuator imparts a nodding motion to the head. Displacement data is captured via a Siemens 3T Allegra head-only scanner (Siemens Medical Solutions; Erlangen, Germany). A multi-shot MRE sequence utilizing spiral readout gradients [21] with periods matching that of the applied shear wave was developed to reduce errors during the inversion step. In total, the imaging volume comprised twenty axial slices of $2 \mathrm{~mm}$ thickness covering the ventricles, corpus callosum and corona radiata resulting in a $2 \times 2 \times 2 \mathrm{~mm}^{3}$ isotropic spatial resolution for the reconstructed mechanical properties.

A non-linear inversion (NLI) algorithm [19] was applied to estimate the material properties from the measured dusplacement data. The inversion is performed by minimizing the function

$$
\Phi(\theta)=\sum_{i=1}^{N}\left(u_{i}^{c}(\theta)-u_{i}^{m}\right)\left(u_{i}^{c}(\theta)-u_{i}^{m}\right)^{*}
$$

by iteratively updating the material property description, given by $\theta$. Here, $u_{i}^{m}$ is the measured displacement amplitude at the location $i ; u_{i}^{c}(\theta)$ is the computed displacement amplitude sampled at the same point; and * indicates the complex conjugate.

Following the development in [22], a Rayleigh damping model is used to represent the material response of brain tissue under time-harmonic conditions. The motion amplitude field $u$, was calculated from Navier's equation for an inhomogeneous, locally isotropic linear elastic medium

$$
\nabla \cdot G\left(\nabla u+\nabla u^{T}\right)+\nabla(\lambda \nabla \cdot u)=\rho \omega^{2} u
$$

where $\lambda$ is the first Lamé parameter; $G$ is the second Lamé parameter, or shear modulus; $\rho$ is the density; and $\omega$ is the activation frequency. The Rayleigh damping model introduces the complex-valued shear modulus and density to account for attenuation related to both elastic and inertial forces - where the 
imaginary shear modulus includes damping effects due to inertial forces, while the imaginary part of the density includes damping related to inertial forces. Including inertial damping effects - something that commonly used viscoelastic models do not include - allows for better characterization of material response when performing the inversion. We use the notation $G^{\prime}$ and $G^{\prime \prime}$ to denote the storage and loss shear moduli respectively, which are the real and imaginary part of the complex valued shear modulus

$$
G=G^{\prime}+i G^{\prime \prime}
$$

where $i$ is the imaginary unit. Due to the nearly incompressible nature of brain tissue, we assume that $\lambda$ is very large compared to the shear modulus. The damping ratio

$$
\xi:=G^{\prime \prime} / 2 G^{\prime}
$$

can be determined as well which physically describes the level of motion attenuation in the tissue.

The distribution of storage and loss moduli within the white matter in the imaging resolution $\left(2 \times 2 \times 2 \mathrm{~mm}^{3}\right)$ is presented in Figure 1 for the coronal, sagittal and horizontal planes. The relative stiffness of the corpus callosum and corona radiata can be clearly observed. The average values and the standard deviation for the global white and gray matter is presented in Table 1 as well as those for the corpus callosum and corona radiata.
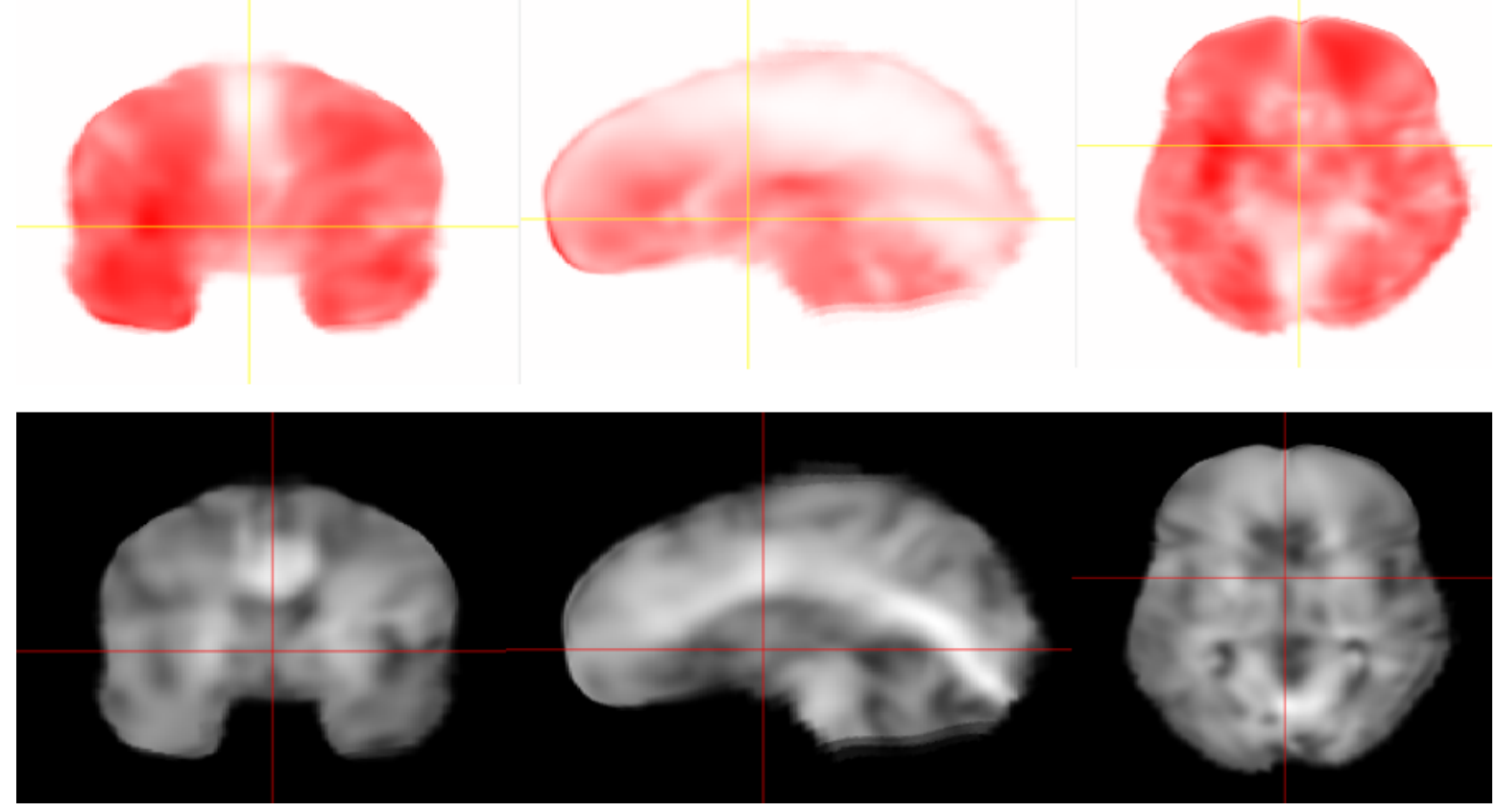

Figure 1. Distribution of Loss (top) and Storage (bottom) modulus in the FE model. Darker regions indicate higher magnitude of shear modulus. 
Table 1. Average values and standard deviations for different tissues within the model: gray Matter (GM), White Matter (WM), Corpus Callosum (CC), Corona Radiata (CR)

\begin{tabular}{|c|c|c|c|c|}
\hline & GM & WM & CC & CR \\
\hline $\mathbf{G}^{\prime} \mathbf{( k P a )}$ & $2.02 \pm 0.09$ & $2.66 \pm 0.30$ & $3.09 \pm 0.39$ & $2.78 \pm 0.37$ \\
\hline $\mathbf{G}^{\prime \prime} \mathbf{( k P a )}$ & $1.04 \pm 0.12$ & $1.54 \pm 0.15$ & $1.23 \pm 0.26$ & $1.97 \pm 0.12$ \\
\hline $\boldsymbol{\xi}$ & $0.32 \pm 0.03$ & $0.31 \pm 0.03$ & $0.23 \pm 0.07$ & $0.37 \pm 0.05$ \\
\hline
\end{tabular}

\subsection{Finite Element Mesh Generation}

In addition to the MRE image acquisition, T1-weighted anatomical images with a resolution of $1 \times 1 \times 1 \mathrm{~mm}^{3}$ are generated for the purposes of mesh segmentation. Image voxels are directly converted to eight-noded hexahedral elements through a custom code, thus preserving the same spatial resolution as the MRI scans. The resulting mesh consists of approximately 2.2 million elements. The mesh is segmented into the following tissue types: scalp, skull, cerebrospinal fluid (CSF), gray matter and white matter. Each element is assigned a different material definition based on the results of the segmentation, as detailed in section 2.3. Features that are below the imaging resolution such as membranes, blood vessels, bridging veins, and draining sinuses are excluded from the model. Since T2-weighted scans were not collected, the segmentation of the interfaces between tissue types is negatively affected. We perform a mesh smoothing operation at these interfaces to minimize this effect. We utilize a volume-preserving Laplacian smoothing algorithm as outlined in [1]. Smoothing has the added benefit of eliminating numerical artifacts that may manifest from jagged edges along interfaces. We ensure traction and displacement continuity at all material interfaces.

Our MRI voxel-based approach produces meshes which more realistically model the complicated folding structure of the cerebral cortex (i.e. gyri and sulci) as well as the differentiation of gray and white matter of brain tissues. Additionally, in order to accurately resolve the shear wave motion within the brain, the mesh must be sufficiently refined [11]. For example, using the typical speeds of shear wave propagation in brain tissue, $c_{T} \approx 5 \mathrm{~m} / \mathrm{s}$, and the frequency of the vibration as $50 \mathrm{~Hz}$, we arrive at a minimum element size of $10 \mathrm{~mm}$ (using a conservative choice of 10 elements per wavelength [23]). For vibrations of higher frequency or more transient loading (such as the cases for impact loads), this requirement is even more stringent.

\subsection{Material Properties}

As discussed above, we assign different material definitions to each voxel based on the result of the mesh segmentation. Due to the presence of highly oriented tracts of myelin-sheathed axonal fibers in white matter, significant heterogeneity exist in this region, especially within the corpus callosum (CC) and corona radiata (CR). On the other hand, gray matter is made up of cell bodies and supporting vascular networks that can be assumed to be isotropic and homogeneous $[13,24]$. This assumption 
Table 2. Material properties of different tissues used in the FE model. For the heterogeneous white matter, only the average values of $G_{0}$ and $G_{\infty}$ are presented, denoted by the "*".

\begin{tabular}{|c|c|c|c|c|c|}
\hline Tissue & Mass Density $\left(\mathrm{kg} / \mathrm{m}^{3}\right)$ & Bulk Modulus K (Pa) & $\begin{array}{l}\text { Short Term Shear } \\
\text { Modulus } G_{0}(\mathrm{~Pa})\end{array}$ & $\begin{array}{l}\text { Long Term Shear } \\
\text { Modulus } G_{\infty}(\mathrm{Pa})\end{array}$ & Decay Factor $\beta\left(\sec ^{-1}\right)$ \\
\hline $\begin{array}{c}\text { gray } \\
\text { matter }\end{array}$ & 1040 & $2.19 \mathrm{E}+9$ & $3.4 \mathrm{E}+4$ & $6.4 \mathrm{E}+3$ & 400 \\
\hline CSF & 1004 & $2.19 \mathrm{E}+7$ & \multicolumn{2}{|c|}{$5.0 \mathrm{E}+4$} & N/A \\
\hline
\end{tabular}

allows for the MRE imaging to be performed over a manageable acquisition volume. As such, we allow for only the white matter to have material heterogeneity whereas other tissues are assumed to be homogeneous.

For homogeneous tissues, the choice of material properties is based on data available in the literature, presented in Table 2, and is the same as that presented in our earlier works [1,3]. The skull is assumed to be linear elastic and modelled as a single-layer structure with homogenized properties given in [25]. We assume a linear viscoelastic material model for the shear response of the brain tissues. The standard linear solid model is chosen for its simplicity where the shear relaxation modulus is given by

$$
G(t)=G_{\infty}+\left(G_{0}-G_{\infty}\right) e^{-\beta t}
$$

Here, $G_{0}$ is the short-term shear modulus, $G_{\infty}$ is the long-term shear modulus and $\beta$ is the decay factor. For the white matter, we assume that only $G_{0}$ and $G_{\infty}$ are heterogeneous with averages scaled to match the values taken from literature [26]. The mass density, bulk modulus and decay factor are maintained to be homogeneous. For the material model for the CSF, we have the choice of three models, previously presented in [3]: incompressible elastic, viscoelastic, and fluid-like elastic using an equation of state model. We determined that the choice of CSF affects the shear wave propagation within the brain while the peak pressure is not significantly altered. For the first iteration of our heterogeneous model, we choose the most commonly utilized model - nearly incompressible elastic model with bulk modulus to be very large compared to the shear modulus, with values taken from [27].

\subsection{Boundary Conditions}

We consider two extreme assumptions for the head-neck junction, free and fixed boundary conditions mostly following previous work in [1]. The free boundary condition allows for predominantly rectilinear motion under frontal impacts while rotational motion cannot be represented. We consider the fixed boundary condition as the other extreme case where the nodes around an area of the foramen magnum are fully constrained. Research reported in [1] showed that the responses from these two boundary conditions bound the experimental response. 


\subsection{Experimental Verification}

We use Nahum et al. linear impact experiments [28] to verify the accuracy of our model. In the experiment, a seated cadaver was impacted by a cylindrical impactor with a constant velocity of $9.94 \mathrm{~m} / \mathrm{s}$. The impact was along the specimen's mid-sagittal plane in an anterior-posterior direction. The skull was rotated as depicted in Figure 2a. The resultant input force time history is given in Figure 2b. Intracranial pressure history was recorded during the impact event. The results of this comparison are presented in the next section. Our homogeneous model was also verified using tagged MRI and harmonic phase (HARP) imaging analysis technique in [2] where displacement time history from head-drop experiments is compared to numerical results. Finally, we directly compare displacement data during impact utilizing experimental data from Hardy et al. brain translational motion experiment [29,30]. Brain motion was captured using neutral density targets (NDT) under linear accelerations ranging from 38 to $291 \mathrm{~g}$.

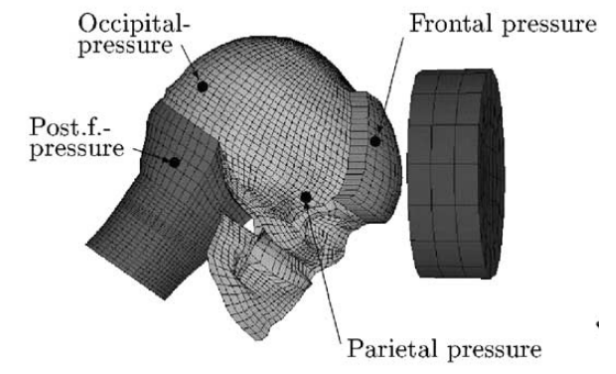

(a)

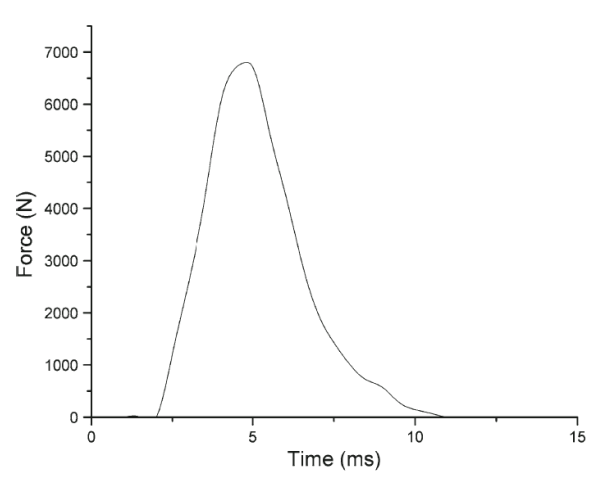

(b)

Figure 2. (a) Impact location in Nahum et al.'s experiments (adapted from [31]). (b) Input force time history (adapted from [1]).

\section{Results and Discussion}

\subsection{Simulation of Impact}

Blunt forces are applied to the head in the frontal region. The recorded impact force from the frontal cadaveric impact experiment conducted by Nahum, as discussed in the preceding section, is directly applied to the model in the form of a distributed load with the peak pressure input of 4.37 $\mathrm{MPa}$. The impact pulse lasts about $9 \mathrm{~ms}$ and the simulation is run for $15 \mathrm{~ms}$. The base of the skull is not constrained to allow the rotation of the model under impact. The finite element simulations are carried out using Abaqus/Explicit.

The pressure-time history for the Nahum loading is presented in Figure 3 at the coup impact site. We plot the results for both the homogeneous and heterogeneous model. At the coup site, the model with free boundary condition predicts tensile pressure in nearly the whole duration with a peak 
pressure of $180 \mathrm{KPa}$ at $5 \mathrm{~ms}$; the model with fixed boundary condition shows variations of compression and tension and the peak pressure reached is $80 \mathrm{KPa}$. Hence, the model with free boundary condition gives better correlation of coup pressure with experimental results. At the contrecoup site, the model with free boundary condition predicts mostly tensile pressure for the $15 \mathrm{~ms}$ simulation and the response for the first $6 \mathrm{~ms}$ is in good agreement with the test data. However, the transition from tension to compression found in the cadaver experiment for the contrecoup site is replicated better by the model with fixed boundary condition. The maximum and minimum contrecoup pressures obtained by the simulations are $65 \mathrm{KPa}$ and $60 \mathrm{KPa}$, respectively. For both cases, the heterogeneous model predicts responses more closely matching the experimental results, indicating that the inclusion of heterogeneity leads to a more realistic model.

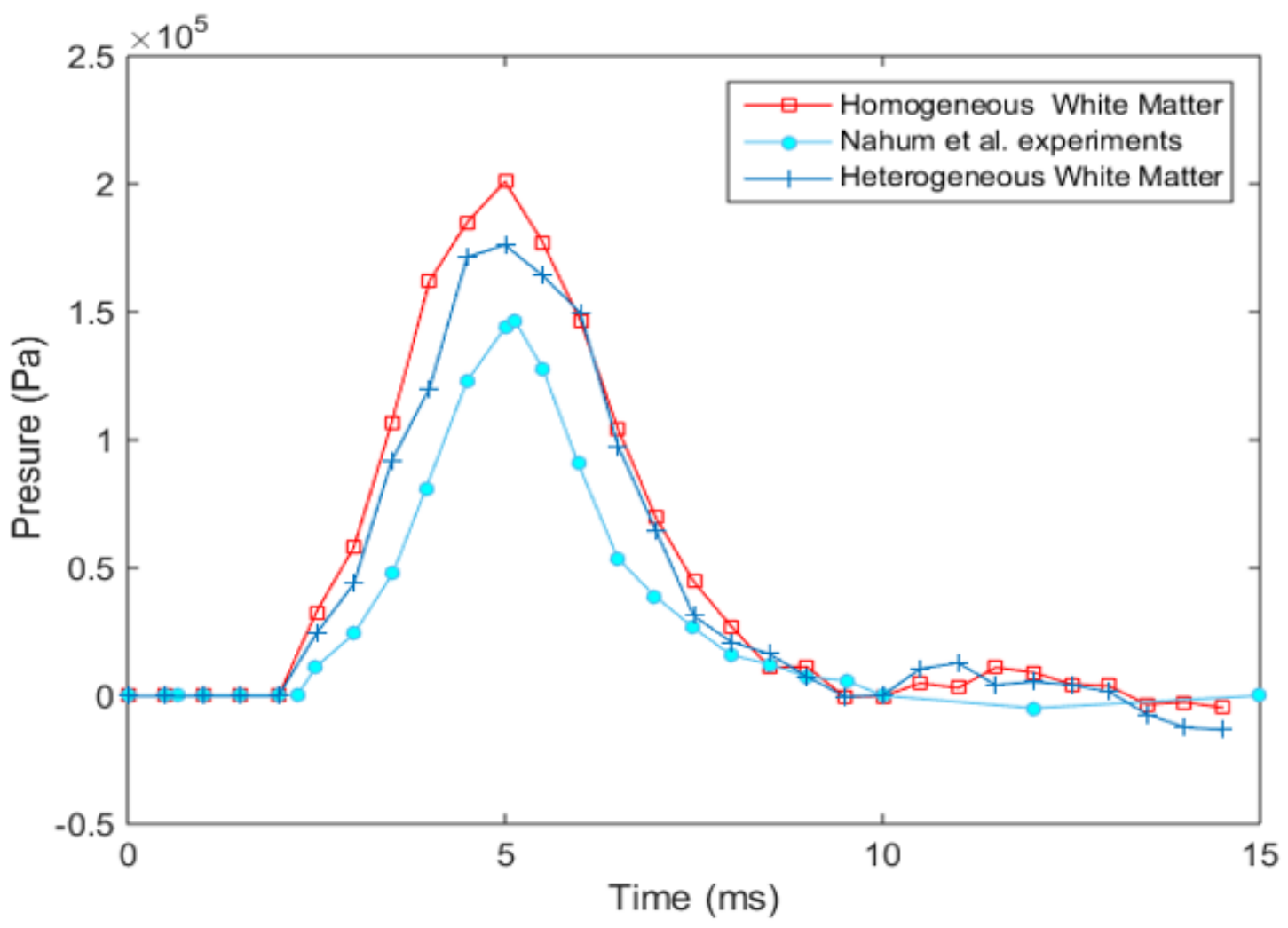

Figure 3. Comparison of heterogeneous model with experimental data from [28]. We find that the heterogeneous model exhibits response closer to the Nahum data.

We next compare the displacement response to the C383-T1 impact from Hardy et al. experiments [29]. We plot the response for relative displacements for the $x$ - and $y$-directions for two NDTs each in the anterior (A) and posterior (P) positions, see Figures 4 and 5 . We find good agreement between our model and the experimental data as shown. Additionally, we have previously validated our homogeneous model using tagged MRI and HARP imaging analysis techniques in [2] which allows comparisons of displacement fields for the entire cerebrum in vivo. 

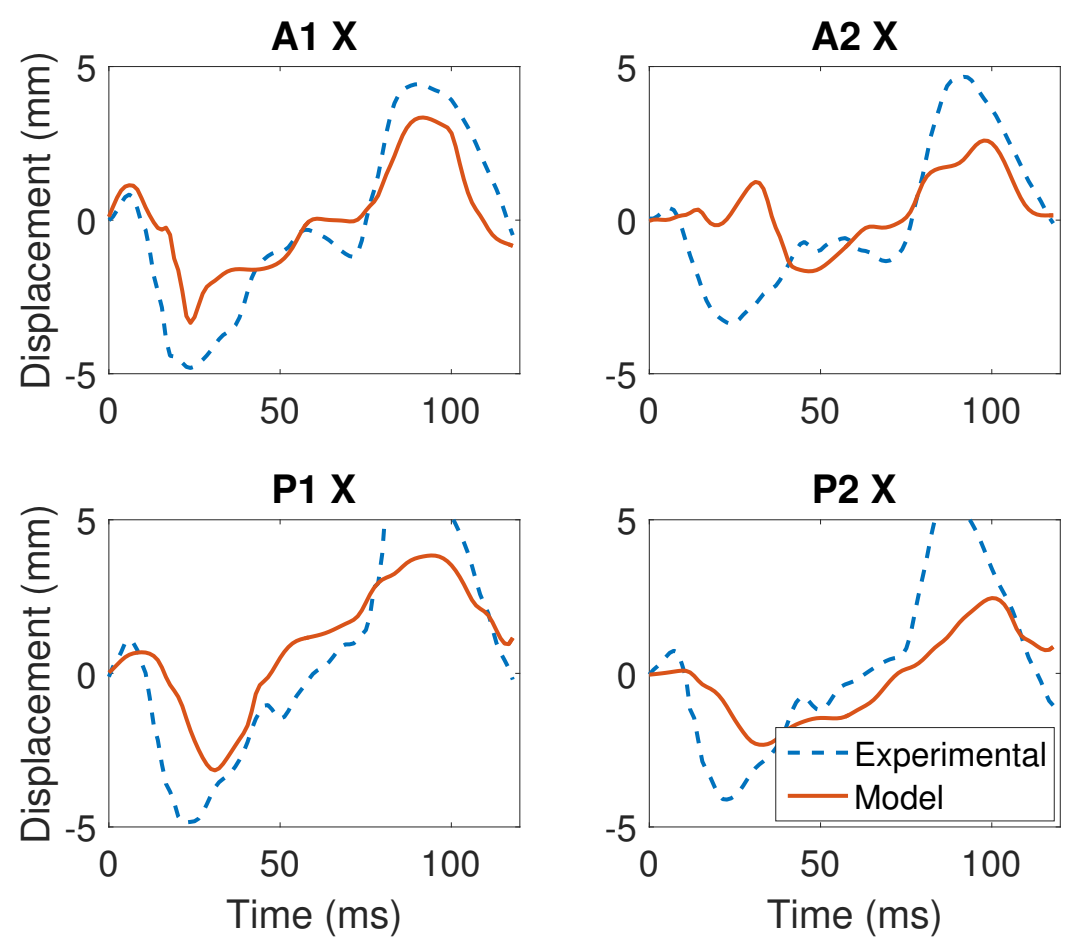

Figure 4. Comparison of displacements in the $x$-direction for two positions in the anterior (A) and posterior $(\mathrm{P})$ to Hardy C83-T1 experiment

We plot the contours of the von Mises stress distribution on the sagittal plane due to the frontal impact in Figure 6. The spherically convergent structure of the shear wave is observed quite clearly. As the wave travels inwards towards the center of the brain, it attenuates and eventually dissipates before traveling a large distance towards the center of the brain. Reflections of wave due to scattering from heterogeneous white matter structures can also be observed at later times.

Next, we consider three distinct points along the sagittal plane, as depicted in Figure 7. The points are chosen within regions of strong heterogenities due to the presence of highly aligned axon tracts, such as the corpus callosum and corona radiata. The differences in mechanical properties of these regions are given in Table 3. We see that the material phases at these points are relatively stiffer than the corresponding points in the homogeneous model. As a result, the response in Figure 7 is affected accordingly. We find that the difference in peak pressure response is proportional to the difference in shear stiffness between the homogeneous and heterogeneous models. However, the time at which these events occur is not significantly affected. This indicates that the pressures in regions of high stiffness within the brain are over-estimated in the homogeneous models. In summary, relative to the MRI-based model, the new MRE-based heterogeneous model more accurately predicts the local response within the white matter by taking into account the differences in tissue stiffness of local white matter structures. 

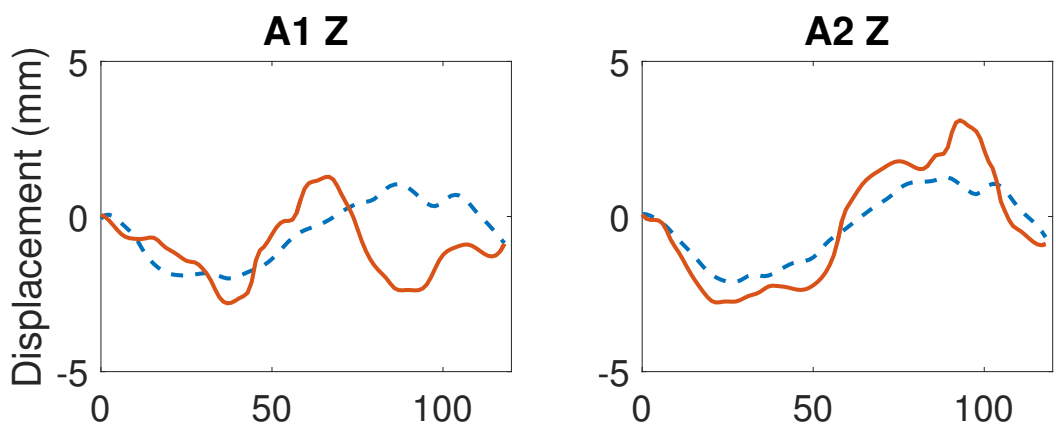

P1 Z

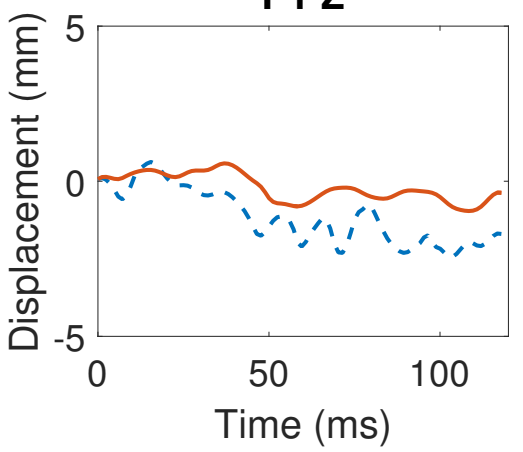

P2 Z

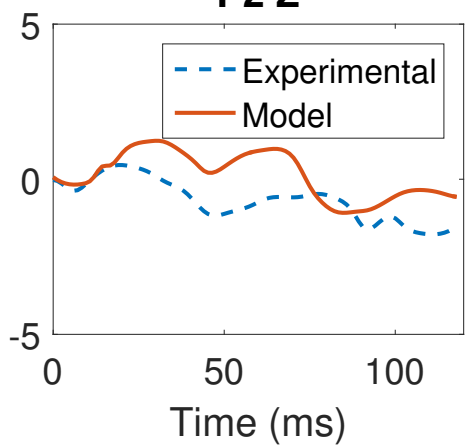

Figure 5. Comparison of displacements in the $z$-direction for two positions in the anterior (A) and posterior $(\mathrm{P})$ to Hardy C83-T1 experiment

A few points are in order regarding the qualitative differences between the shear modulus of different regions in our model. Globally, the white matter is found to be approximately $32 \%$ stiffer than the gray matter. In general, the white matter properties in local regions differ significantly from the average ones. For instance, the storage modulus $G^{\prime}$ is significantly lower in the rest of the white matter than within the corpus callosum and the corona radiata. This is quite logical given the fact that the corpus callosum contains highly oriented, tightly packed axon tracts. The corpus callosum in turn is stiffer than the corona radiata, again evident from the composition of the corona radiata which contains axon fibers that fan out and are not as highly aligned as the corpus callosum. Additionally, while both white and gray matter have similar values of damping ratio, $\xi$ (which reflects the amount of motion attenuation within the tissue), the corpus callosum has a lower value while the corona radiata has a higher value. This can be explained by examining the microstructure of each of these regions. Experiments by Guo et al. [32] demonstrated that damping ratio (and thus the attenuation) in soft tissue composites increases as the number of cross-links between fibers increases. The corona radiata consists of fibers arranged in a grid-like pattern [33] with a large number of cross-links. These crossings do not exist in the corpus callosum, offering a possible explanation for the distribution of $\xi$.

Since mechanical measures from MRE and diffusivity measures from diffuse tensor imaging (DTI) are essentially measures of the distribution of axon tracts within white matter, a natural question arises here - what difference is there between our heterogeneous (yet isotropic) model and the more common 

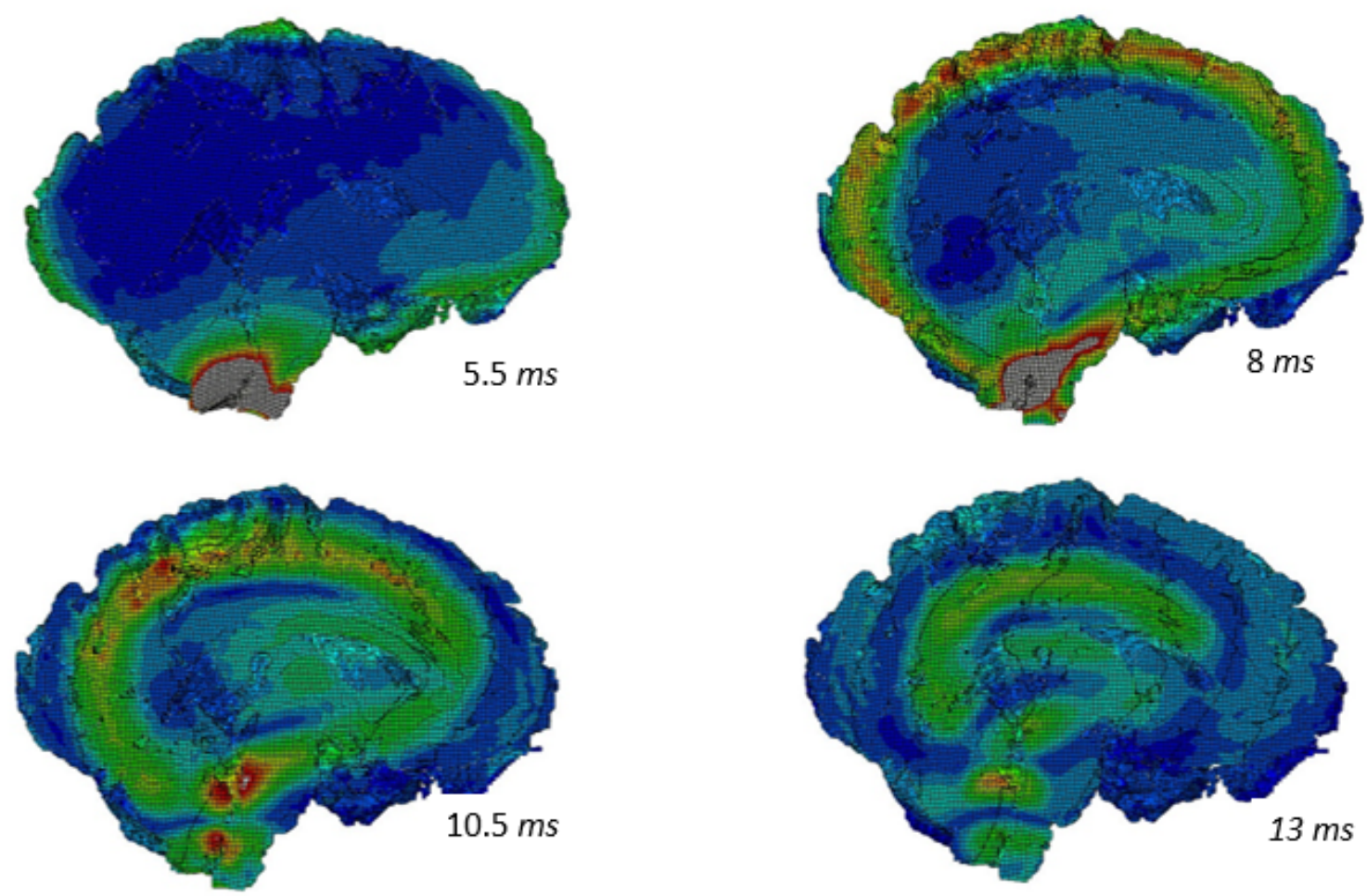

Figure 6. Shear wave propagation due to frontal impact. Notice the attenuation of the wavefront as time progresses.

Table 3. Difference of material properties and peak pressure response for three distinct points (indicated in Figure 7) along the sagittal plane within the white matter.

\begin{tabular}{|c|c|c|}
\hline Location & \% Difference in Shear Modulus $\left(G_{\infty}\right)$ & \% Difference in Peak Pressure \\
\hline 1 & 13.34 & -7.86 \\
\hline 2 & 28.62 & -47.25 \\
\hline 3 & 15.48 & -20.75 \\
\hline
\end{tabular}

anisotropic FE models where fiber anisotropy is determined from DTI scans? Many such examples of the latter exist in the literature: for instance in [34-37]. Johnson et al. [13] performed both MRE and DTI measurements on a group of seven volunteers to determine the correlation of mechanical and diffusivity measures within the corpus callosum and corona radiata. They determined that MRE and DTI measures correlate well with each other within the corpus callosum - not surprising since they are both sensitive to the underlying tissue microstructure. They hypothesize that these measures are highly dependent on axon diameter since larger axons provide greater structural rigidity to the tissue [38]. Within the corona radiata, however, the correlation is not as significant. The corona radiata comprises fiber tracts that fan towards the cortex and contain numerous crossings [33] which are not captured well by DTI [39]. This has been hypothesized as the reason for the poor correlation within the corona radiata. More work is needed to determine the differences between these two methods when used within FE models. 
(1)

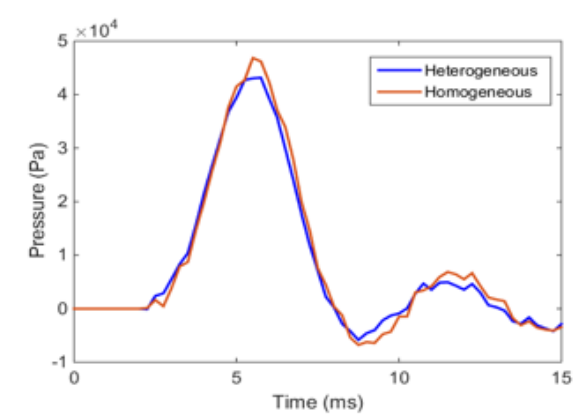

(2)

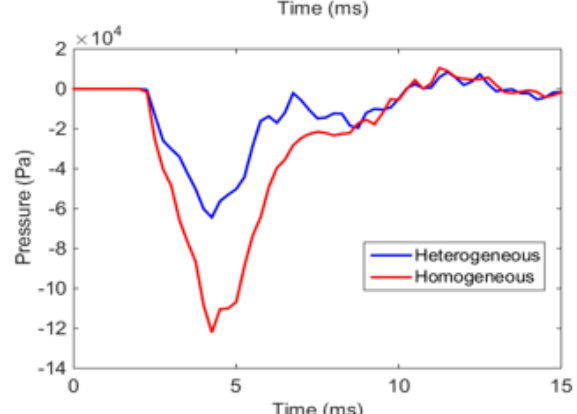

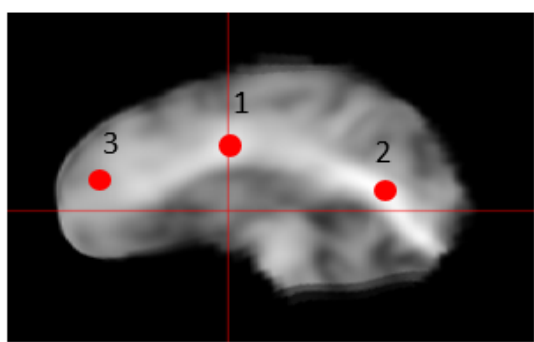

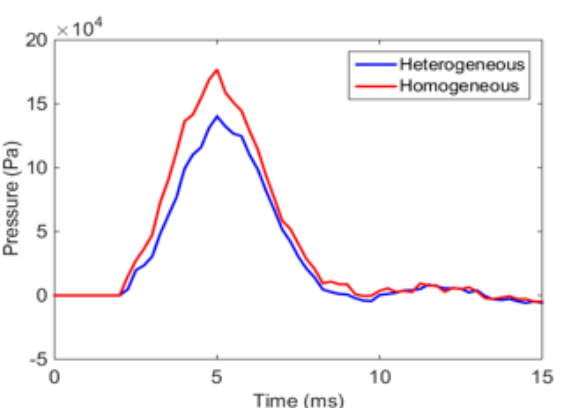

Figure 7. Comparison of pressure at three points on the sagittal plane. The difference in the heterogeneous and homogeneous models is most evident in regions of high relative stiffness.

\subsection{Stochastic Wave Propagation}

The highly heterogeneous structure of the brain tissue introduces wave scattering that competes with wave amplification due to spherically convergent implosion. Following the development in [40], we investigate this effect by considering the theory of wavefronts. For the case of one-dimensional wave motion, we assume that a compressive load produces a shock wavefront that propagates from a disturbed domain to an undisturbed one with a speed $c_{T}$. The initial conditions can be given as $u(x, 0)=u_{, t}(x, 0)=0 ; \tau(0, t)=-\tau_{0} H(t)$ where $H$ is the Heaviside function.

First, assuming a plane wave in a homogeneous medium, we have the dynamic compatibility condition $[\tau]=-\rho c_{T}[u, t]$ in the $(x, t)$ - plane, where the notation $[f]$ denotes the discontinuity in function $f$ across the boundary of two material domains 1 and 2, $\sigma$ is the shear stress, $\rho$ is the mass density, $u$ is the displacement normal to the direction of wave motion, and $c_{T}$ is the transverse wave speed. The linear viscoelastic stress-strain relation for a process that started at time $t=t_{0}^{+}$is

$$
\tau(t)=G(0) \varepsilon(t)+\int_{t_{0}^{+}}^{t} G_{, t}(t-s) \varepsilon(s) d s=G(0) \frac{1}{c_{1}} u_{, x}(t)+\int_{t_{0}^{+}}^{t} G_{, t}(t-s) \frac{1}{c_{1}} u_{, x}(s) d s
$$

where $\varepsilon$ is the shear strain. We can derive the relationship for the wavespeed as $c_{T}=\sqrt{G(0) / \rho}$, where $G(0)$ is the glassy modulus. Following the derivation in [40], we obtain the equation governing the evolution of the discontinuity of $\tau$ at the wavefront as: 


$$
\frac{d_{T}}{d t}[\tau]=\frac{1}{2} \frac{G, t(0)}{G(0)}[\tau]
$$

Given the initial conditions given above, the solution of equation 3.2 can is given as

$$
[\tau]=-\tau_{0} \exp \left[\frac{1}{2} \frac{G, t(0)}{G(0)}[\tau]\right]
$$

Given that $G, t(0) \leq 0$, and $G(0)>0$, the stress jump exhibits exponential attenuation and has a tendency for blow-up as $r \rightarrow 0$. As our simulations here and in [3] show, the attenuation is strong enough so that the impact-generated imploding waves do not seem to blow up into a singularity at the head center.

The impact gives rise not only to a fast pressure wave, but also to a slower shear wave. Due to its relatively low shear modulus, brain tissue deforms more readily to shearing forces. Therefore, the shear wave has the potential to be more damaging. The spherically convergent shear wave patterns are observed even in the case of homogeneous material description, c.f. [1]. The attenuation of the pressure along the sagittal plane for both the homogeneous and heterogeneous models are presented in Figure 8. It is clear that the attenuation is greater in the heterogeneous model as predicted.

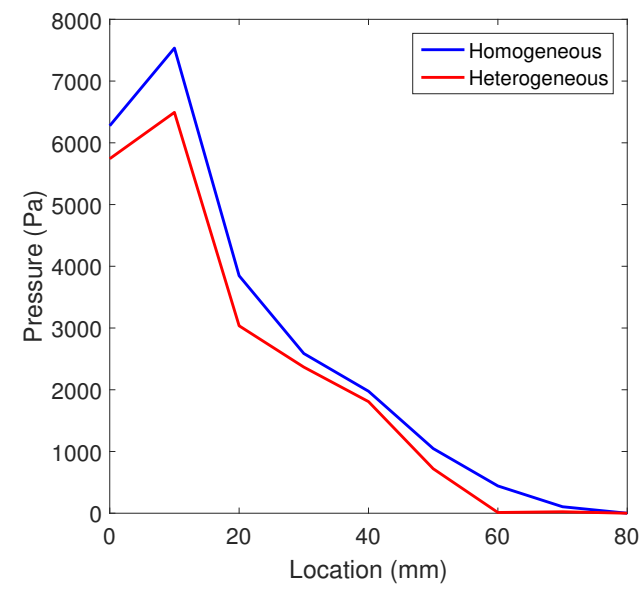

(a)

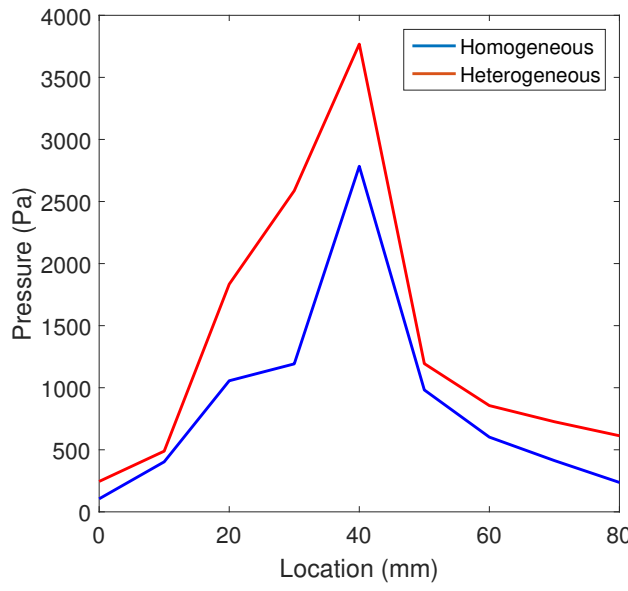

(b)

Figure 8. Comparison of attenuation of pressure wave along the sagittal plane between homogeneous and heterogeneous model. (a) At $3 \mathrm{~ms}$ and (b) at $7 \mathrm{~ms}$.

\subsection{Limitations}

We list here a few limitations in our methodology, some of which we hope to improve in future works. Features that are below the imaging resolution such as membranes (in particular, the falx cerebri and tentorium), blood vessels, bridging veins, and draining sinuses are excluded from the current model. However, we argue that the inclusion of heterogeneous MRE-derived parameters in white matter tissue alleviates some of the drawbacks of excluding these features. Additionally, 
since the MRE inversion was performed assuming locally linear viscoelastic material model, we have chosen to utilize linear viscoelasticity for white and grey matter. Research has shown that the the shear modulus of the brain tissue is much lower than that used in this paper [41]. We aim to perform an optimization study to tune the values chosen here to better match experimental results.

\section{Conclusion}

We present a high-resolution, voxel-based heterogeneous finite element head model to study transient wave dynamics during traumatic brain injury. We utilize heterogeneous shear modulus determined using a nonlinear inversion technique from MRE experiments performed by [13]. While many FE models employ homogenized or averaged, mechanical properties to approximate constitutive relations of brain tissues, our approach allows us to investigate response due to local structures within the white matter. We note that both the corpus callosum and corona radiata are significantly stiffer than overall white matter, with the corpus callosum exhibiting greater stiffness and less viscous damping than the corona radiata. These differences are explained by examining the organizational and compositional characteristics of each structure. Incorporating this heterogeneity in our model affects wave propagation within the cerebrum and yields results that more closely match experimental results. We find that local variations in stiffness affect the local mechanical response. For instance, intracranial pressure magnitude following an impact is lower in regions of high local stiffness.

The unique architecture of the human head, consisting of the hard solid skull, the membranes, CSF, and the viscoelastic brain core, leads to partial conversion of the pressure impact into a shear wave converging towards head's center. However, the shear wave attenuates as it converges inward due to (1) wave damping due to brain tissue viscoelasticity and (2) the heterogeneous brain structure which introduces extra wave scattering. The magnitude of this attenuation is greater for the heterogeneous material model.

\section{References}

1. Chen, Y.; Ostoja-Starzewski, M. MRI-based finite element modeling of head trauma: spherically focusing shear waves. Acta Mechanica 2010, 213, 155-167.

2. Chen, Y.; Sutton, B.; Conway, C.; Broglio, S.P.; Ostoja-Starzewski, M. Brain Deformation Under Mild Impact: Magnetic Resonance Imaging-Based Assessment and Finite Elmenent Study. International Journal of Numerical Analysis and Modeling, Series B 2012, 3, 20-35.

3. Madhukar, A.; Chen, Y.; Ostoja-Starzewski, M. Effect of cerebrospinal fluid modeling on spherically convergent shear waves during blunt head trauma. International Journal for Numerical Methods in Biomedical Engineering 2017, 33.

4. Taylor, C.A.; Bell, J.M.; Breiding, M.J.; Xu, L. Traumatic Brain Injury-Related Emergency Department Visits, Hospitalizations, and Deaths-United States, 2007 and 2013. Morbidity and mortality weekly report. Surveillance summaries (Washington, DC: 2002) 2017, 66, 1-16.

5. Gennarelli, T.A.; Thibault, L.E.; Adams, J.H.; Graham, D.I.; Thompson, C.J.; Marcincin, R.P. Diffuse axonal injury and traumatic coma in the primate. Annals of Neurology 1982, 12, 564-574. 
6. Gentry, L.R.; Godersky, J.C.; Thompson, B.; Dunn, V.D. Prospective comparative study of intermediate-field MR and CT in the evaluation of closed head trauma. American journal of Roentgenology 1988, 150, 673-682.

7. Gentry, L.R.; Godersky, J.C.; Thompson, B. MR imaging of head trauma: review of the distribution and radiopathologic features of traumatic lesions. American Journal of Roentgenology 1988, 150, 663-672.

8. Ng, H.; Mahaliyana, R.; Poon, W. The pathological spectrum of diffuse axonal injury in blunt head trauma: assessment with axon and myelin stains. Clinical Neurology and Neurosurgery 1994, 96, 24-31.

9. Arfanakis, K.; Haughton, V.M.; Carew, J.D.; Rogers, B.P.; Dempsey, R.J.; Meyerand, M.E. Diffusion tensor MR imaging in diffuse axonal injury. American Journal of Neuroradiology 2002, 23, 794-802.

10. Arbogast, K.B.; Margulies, S.S. Material characterization of the brainstem from oscillatory shear tests. Journal of Biomechanics 1998, 31, 801-807.

11. Madhukar, A.; Ostoja-Starzewski, M. Finite element methods in human head impact simulations: a review. Annals of Biomedical Engineering 2019, pp. 1-23.

12. Chatelin, S.; Constantinesco, A.; Willinger, R. Fifty years of brain tissue mechanical testing: from in vitro to in vivo investigations. Biorheology 2010, 47, 255-276.

13. Johnson, C.L.; McGarry, M.D.; Gharibans, A.A.; Weaver, J.B.; Paulsen, K.D.; Wang, H.; Olivero, W.C.; Sutton, B.P.; Georgiadis, J.G. Local mechanical properties of white matter structures in the human brain. Neuroimage 2013, 79, 145-152.

14. Muthupillai, R.; Lomas, D.; Rossman, P.; Greenleaf, J.F.; Manduca, A.; Ehman, R.L. Magnetic resonance elastography by direct visualization of propagating acoustic strain waves. science 1995, 269, 1854-1857.

15. Sack, I.; Streitberger, K.J.; Krefting, D.; Paul, F.; Braun, J. The influence of physiological aging and atrophy on brain viscoelastic properties in humans. PloS one 2011, 6, e23451.

16. Murphy, M.C.; Huston III, J.; Jack Jr, C.R.; Glaser, K.J.; Manduca, A.; Felmlee, J.P.; Ehman, R.L. Decreased brain stiffness in Alzheimer's disease determined by magnetic resonance elastography. Journal of magnetic resonance imaging 2011, 34, 494-498.

17. Murphy, M.C.; Huston, J.; Glaser, K.J.; Manduca, A.; Meyer, F.B.; Lanzino, G.; Morris, J.M.; Felmlee, J.P.; Ehman, R.L. Preoperative assessment of meningioma stiffness using magnetic resonance elastography. Journal of neurosurgery 2013, 118, 643-648.

18. Boulet, T.; Kelso, M.L.; Othman, S.F. Microscopic magnetic resonance elastography of traumatic brain injury model. Journal of neuroscience methods 2011, 201, 296-306.

19. Van Houten, E.E.; Miga, M.I.; Weaver, J.B.; Kennedy, F.E.; Paulsen, K.D. Three-dimensional subzone-based reconstruction algorithm for MR elastography. Magnetic Resonance in Medicine: An Official Journal of the International Society for Magnetic Resonance in Medicine 2001, 45, 827-837.

20. Johnson, C.L.; McGarry, M.D.J.; Van Houten, E.E.W.; Weaver, J.B.; Paulsen, K.D.; Sutton, B.P.; Georgiadis, J.G. Magnetic resonance elastography of the brain using multishot spiral readouts with self-navigated motion correction. Magnetic Resonance in Medicine 2013, 70, 404-412. doi:10.1002/mrm.24473.

21. Glover, G.H. Simple analytic spiral K-space algorithm. Magnetic Resonance in Medicine 1999, 42, 412-415. doi:10.1002/(SICI)1522-2594(199908)42:2<412::AID-MRM25>3.0.CO;2-U.

22. McGarry, M.D.J.; Van Houten, E.E.W. Use of a Rayleigh damping model in elastography. Medical $\mathcal{E}$ Biological Engineering E Computing 2008, 46, 759-766.

23. Bradshaw, D.; Morfey, C. Pressure and shear response in brain injury models. Proceedings of the 17th international technical conference on the enhanced safety of vehicles, Amsterdam, The Netherlands, 2001.

24. Prange, M.; Margulies, S. Regional, directional, and age-dependent properties of the brain undergoing large deformation. Journal of Biomechanical Engineering 2002, 124, 244-252. doi:10.1115/1.1449907.

25. Khalil, T.B.; Hubbard, R.P. Parametric study of head response by finite element modeling. Journal of Biomechanics 1977, 10, 119-132.

26. Zhang, L.; Yang, K.H.; King, A.I. A proposed injury threshold for mild traumatic brain injury. Journal of Biomechanical Engineering 2004, 126, 226-236.

27. Zhou, C.; Khalil, T.B.; King, A.I. A new model comparing impact responses of the homogeneous and inhomogeneous human brain. Technical report, SAE Technical Paper, 1995.

28. Nahum, A.M.; Smith, R.; Ward, C.C. Intracranial pressure dynamics during head impact. Technical report, SAE Technical Paper, 1977. 
29. Hardy, W.N.; Foster, C.D.; Mason, M.J.; Yang, K.H.; King, A.I.; Tashman, S. Investigation of head injury mechanisms using neutral density technology and high-speed biplanar X-ray. Stapp Car Crash Journal 2001, 45, 337-368.

30. Hardy, W.N.; Mason, M.J.; Foster, C.D.; Shah, C.S.; Kopacz, J.M.; Yang, K.H.; King, A.I.; Bishop, J.; Bey, M.; Anderst, W.; others. A study of the response of the human cadaver head to impact. Stapp car crash journal 2007, 51, 17.

31. Kleiven, S.; von Holst, H. Consequences of head size following trauma to the human head. Journal of Biomechanics 2002, 35, 153-160.

32. Guo, J.; Posnansky, O.; Hirsch, S.; Scheel, M.; Taupitz, M.; Braun, J.; Sack, I. Fractal network dimension and viscoelastic powerlaw behavior: II. An experimental study of structure-mimicking phantoms by magnetic resonance elastography. Physics in Medicine E Biology 2012, 57, 4041.

33. Wedeen, V.J.; Rosene, D.L.; Wang, R.; Dai, G.; Mortazavi, F.; Hagmann, P.; Kaas, J.H.; Tseng, W.Y.I. The geometric structure of the brain fiber pathways. Science 2012, 335, 1628-1634.

34. Ji, S.; Zhao, W.; Ford, J.C.; Beckwith, J.G.; Bolander, R.P.; Greenwald, R.M.; Flashman, L.A.; Paulsen, K.D.; McAllister, T.W. Group-wise evaluation and comparison of white matter fiber strain and maximum principal strain in sports-related concussion. Journal of Neurotrauma 2015, 32, 441-454.

35. Giordano, C.; Kleiven, S. Connecting fractional anisotropy from medical images with mechanical anisotropy of a hyperviscoelastic fibre-reinforced constitutive model for brain tissue. Journal of The Royal Society Interface 2014, 11, 20130914.

36. Giordano, C.; Cloots, R.; Van Dommelen, J.; Kleiven, S. The influence of anisotropy on brain injury prediction. Journal of Biomechanics 2014, 47, 1052-1059.

37. Garimella, H.T.; Kraft, R.H. Modeling the mechanics of axonal fiber tracts using the embedded finite element method. International Journal for Numerical Methods in Biomedical Engineering 2017, 33.

38. Arbogast, K.B.; Margulies, S.S. A fiber-reinforced composite model of the viscoelastic behavior of the brainstem in shear. Journal of biomechanics 1999, 32, 865-870.

39. Wedeen, V.J.; Hagmann, P.; Tseng, W.Y.I.; Reese, T.G.; Weisskoff, R.M. Mapping complex tissue architecture with diffusion spectrum magnetic resonance imaging. Magnetic resonance in medicine 2005, 54, 1377-1386.

40. Ostoja-Starzewski, M.; Costa, L. Shock waves in random viscoelastic media. Acta Mechanica 2012, 223, 1777-1788.

41. Miller, K.; Chinzei, K.; Orssengo, G.; Bednarz, P. Mechanical properties of brain tissue in-vivo: experiment and computer simulation. Journal of Biomechanics 2000, 33, 1369-1376. 